\title{
Comportamiento de las comunidades digitales en Twitter durante las elecciones México 2018
}

\author{
Behavior of the digital communities on Twitter during the Mexico \\ elections 2018
}

\author{
Andrade del Cid, P., Flores González, R. y Pablo Contreras, M. 1 \\ Recibido: 19-09-2019 - Aceptado: 14-01-2020 \\ DOI: https://doi.org/10.26441/RC19.1-2020-A2
}

\begin{abstract}
RESUMEN: Con el objetivo de evaluar la importancia de la comunicación digital en la cultura política, se exploró el comportamiento de las comunidades digitales del Twitter durante las elecciones México 2018. La investigación se dividió en dos fases: obtener los tuits de la campaña electoral sobre tres de los candidatos por medio del entorno de programación "R" y mediante filtros el análisis de la base de datos a través de un libro de códigos.
\end{abstract}

Los resultados describen que, de 4664 tuits analizados, el candidato José Antonio Meade (47\%), seguido por Andrés Manuel López Obrador (29\%) y Ricardo Anaya (24\%). Los emisores "ciudadanos comunes" emitieron el $31 \%$ de los tuits analizados, de los cuales el $20 \%$ fueron enlazados con noticias de medios online, mientras que los "periodistas" y "medios de comunicación" sumaron el $40 \%$ del total. La discusión final prevé a la comunicación digital como transformadora o reproductora de la esfera pública.

Palabras clave: big data; análisis de contenido; Twitter; cultura política; México.

\begin{abstract}
In order to assess the importance of political communication in the Mexican political culture, this paper probes into the behavior of digital communities in Twitter during the 2018 Mexico's presidential election.

Research followed a two staged design. First, a large sample of tweets were collected on a daily basis using the package "rtweet" in the R programming environment. Following that, the collected tweets were filtered, cleaned, and a codebook was designed to analyze their contents.

The results show that, out of a sample of 4664 publications, candidate Jose Antonio Meade had the largest number of mentions (47\%), followed by Andrés Manuel López Obrador $(29 \%)$ and Ricardo Anaya (24\%). "Common citizens" issued $31 \%$ of the tweets analyzed, out of which $20 \%$ show links to online news outlets, while journalists and media publications accounted for $40 \%$ of the sampled tweets. The authors consider that these results characterize the observed digital communication practices as either transformations or extensions of the offline public sphere.
\end{abstract}

Keywords: big data; content analysis; Twitter; political culture; Mexico.

\footnotetext{
${ }^{1}$ Patricia Andrade del Cid es Doctora en Ciencias de la Información por la Universidad Complutense de Madrid, Investigadora Nivel 1 del SIN y Fundadora del Observatorio Veracruzano de Medios, adscrito al Centro de Estudios de Opinión y Análisis, de la Universidad Veracruzana. patiandrade59@gmail.com, https://orcid.org/0000-0001-6404-1678

Rubén Flores González es Doctor en Psicología con enfoque en Psicología Política por la Universidad de Guanajuato, Miembro del SNI nivel candidato y Coordinador de la Especialización en Estudios de Opinión de la Universidad Veracruzana. ruben.flores.gn@gmail.com, https://orcid.org/0000-0002-2415-6742
}

Mariangel Pablo Contreras es Licenciada en Publicidad y Relaciones Públicas por la Universidad Veracruzana y Miembro del Observatorio Veracruzano de Medios. mariangelpablo@gmail.com, https://orcid.org/0000-0002-0809-289X 


\section{Introducción}

En los últimos años surgen en nuestro entorno cultural y científico nuevas aproximaciones instrumentales y metodológicas que analizan el dato digital, ya que representan un nuevo paradigma para la comunicación. Tal como lo sugieren Azurmendi, Muñoz y Ortega (2018) "en la sociedad digital del conocimiento, la ciencia de la investigación en comunicación y ciencias sociales se aproxima a disponer de metodologías e instrumentos científicos que proveerán a la ciencia de la comunicación mayor categoría científica" (p.171).

En el contexto de las elecciones 2018 en México, las comunidades digitales tuvieron una gran actividad, por ello este trabajo tiene por objetivo analizar el comportamiento de las comunidades digitales en Twitter, a través de metodologías que se originan en el Big data y que pueden ser examinadas con técnicas del Análisis de Contenido.

Para la construcción del marco teórico esta investigación se pregunta por las características estructurales del lenguaje utilizado en Twitter y la participación de la ciudadanía digital.

Los instrumentos metodológicos pretenden también explorar las interacciones de los usuarios del Twitter, tanto por algunas características de su lenguaje: hashtags, retuits, likes, etc., como por los contenidos que abordan en las conversaciones, y así favorecer la investigación científica de la comunicación.

Para contextualizarlo se presenta un marco teórico que aborda el ámbito de la participación de la ciudadanía digital, cuyas expresiones se integran al ámbito de la comunicación y de la cultura política, considerando a su referente como análisis de representaciones sociales que los provee de identidad y de identidad política.

Además de aportar insumos para el conocimiento de la cultura política, esta propuesta teórica asume que la deliberación que se verifica en esa nueva esfera de lo público- que suele ser emotiva, anónima, viral y a veces falsa -, es uno de los nuevos escenarios en que se desarrolla la política contemporánea.

En el apartado metodológico se describe el procedimiento informático para la extracción de los tuits, el número de mensajes que se analizaron y los elementos empíricos que constan en el "Libro de Códigos" a fin de construir variables y categorías para su estudio. Después se muestran los resultados de ese análisis.

\section{La esfera pública y la ciudadanía digital}

Las redes sociales han demostrado ser un fuerte aliado de la democracia participativa en favor de una esfera pública menos hegemónica y más ciudadana. Esta actividad amplía la esfera pública, porque además de ser un lugar de información promueve la discusión, la contestación, la organización y la lucha política, incluye en ese escenario, la participación de los medios de difusión tradicionales y digitales (Kellner, 2000).

Los medios tradicionales no tuvieron la capacidad para potenciar la democracia participativa, porque, aunque dotaron de un universo simbólico de referencia común a los grupos sociales, no tienen las características de una red social, que basa su interacción en el establecimiento de lazos fuertes - entre cinco y aproximadamente 15 participantes - en lo glocal (Molina 2011). Las redes mantienen una supersocialización, haciendo más densa la comunicación entre redes sociales preexistentes. Sus expresiones suelen semejar a una multitud (Le Bon 1895), al mantener sentimientos de solidaridad colectiva temporal, que aumenta el número de conocidos de cada persona que manifiestan una conducta determinada (Molina, 2011). Esta actividad amplía la esfera pública, porque además 
de ser un lugar de información promueve la discusión, la contestación, la organización y la lucha política, incluyendo en ese escenario la participación de los medios de difusión tradicionales y digitales (Kellner, 2000).

Además, en los medios tradicionales los políticos no recibieron el juicio ciudadano que merecían sus actuaciones, ya que no cabía espacio para la retroalimentación, o simplemente los medios desempeñaron su función de interpretación de lo que les preocupa a los ciudadanos, con el correspondiente sesgo de sus intereses editoriales. En cambio, las redes sociales, implican a la comunicación, promueven la interacción y son la expresión de la ciudadanía que quiere participar política y socialmente. Todas ellas son actitudes que promueven la comunidad e identidad de los grupos sociales.

Natal, Benítez y Ortiz (2014) le llaman ciudadanía digital al corpus evolutivo de la sociedad moderna y las relaciones comunicativas de los individuos por medio de las plataformas tecnológicas disponibles y personalizadas que discuten, se expresan, piensan, reflexionan y se organizan en el ciberespacio.

Esta ciudadanía parece desplazar a los medios informativos en la agenda pública; además, como afirman Pérez, Carabaza, y Cuevas (2013) cada vez es más valorada por los medios tradicionales y digitales:

...Twitter se ha convertido en una herramienta de creciente importancia en las salas de redacción de las empresas periodísticas (...) se trata de un prominente sistema de monitoreo de aquella parte de la agenda pública que se construye en el ciberespacio, y que cuando es retomada por los comunicadores en la prensa, radio y televisión, puede alcanzar un amplio auditorio, incluso entre aquellas personas que carecen de acceso a Internet

Por su agudeza comunicativa es posible prever que, en poco tiempo, la diversidad de plataformas de las redes sociales virtuales, articule los discursos de opinión y promueva nuevas identidades, mediante la equidad participativa, no sin antes, resolver el problema de la brecha digital que conlleva el desarrollo tecnológico (Meneses, 2015).

\section{Cultura política y el Twitter}

Los medios de comunicación han sido los emisores de una parte importante de la información política. Cuando se analiza el contenido de esas informaciones lo que se busca, entre otras cosas, es evidenciar normas y comportamiento de los grupos sociales que consumen cierto (s) tipo de información (es). Al estudiar la comunicación se analiza el comportamiento de los grupos sociales, porque el mensaje está contenido de valores, personajes, acciones y objetos de referencia (Andrade, 2007); esto se advierte a través de las palabras, los símbolos, las argumentaciones, y las particularidades en que se expresan esos contenidos en los distintos lenguajes de los medios.

Las normas y valores proveen identidad política a los grupos sociales, es decir, sentirse parte del grupo significa estar de acuerdo con esa visión del mundo. Por eso el concepto de cultura política se circunscribe a la concepción de un código que orienta el comportamiento político de los "ciudadanos" y no representa a una región o a un país, sino a una manera de entender y vivir la política a través de su - propia - historia. A partir de la realidad histórica de cada nación y/o grupo social, las hipótesis sobre la cultura política son las siguientes:

a) En una sociedad existe una cierta base cultural común sobre ciertos fenómenos políticos.

b) Cualquiera que sea el grado de legitimidad de un régimen político y el nivel de participación ciudadana en la vida política e institucional, la población tendrá ideas favorables o contrarias a la acción de gobierno. 
c) Bajo cualquier régimen -sea éste democrático o autoritario- es posible descubrir ciertas tendencias de opinión en aquellas áreas de la vida política donde exista un mínimo de información directa a partir de la Comunicación Social (Piñuel y Gaytán, 1995).

La cultura política será pues el resultado de la confluencia de orientaciones cognitivas, afectivas y evaluativas hacia el sistema político en general, tanto de sus aspectos políticos y administrativos, como el papel del individuo como miembro activo —o pasivo - de la política (Almond y Verba, 1963).

Tanto las noticias como las interacciones del Twitter que se emiten en campaña política son expresiones de la cultura política de ese lugar, porque emiten información que contiene símbolos -códigos - que logran que ciertos grupos sociales "se identifiquen" y repliquen la información o se congratulen por conocerla (likes).

Las identidades políticas son el lugar simbólico dónde se comparten ideologías, intereses y representaciones; por eso pueden ser utilizadas como referente de la cultura política. Esto significa que las interacciones entre los actores se manifiestan a través de valores, ideologías o intereses de los mismos, utilizando recursos que tengan a su alcance para lo que consideran su beneficio propio.

Por eso, lo que analizamos en las interacciones de las comunidades digitales, son los códigos o representaciones sociales, que se expresan en esos relatos que proceden de la base de la cultura misma; su contenido expresará asuntos que preceden y pertenecen a una comunidad. Durante las campañas políticas los modelos de representación de la cultura política se amplifican dejando que se visibilicen objetos y referencias de distintos grupos y actores sociales, lo que resulta benéfico para el estudio de la disciplina de la comunicación.

De aquellas experiencias se ha comprobado que, así como surgen grupos sui generis con una identidad propia e interés común en el mundo real, emergen grupos que conforman un movimiento en las comunidades digitales, y que también son representativas de intereses u objetivos comunes manteniendo una dinámica organizada, utilizando a las redes sociales como núcleos de cohesión, solidaridad y confianza (Abascal, 2015).

Maldonado (2012) dice que las variables más simbólicas del comportamiento electoral son la intermediación y la discusión política. Cuando la familia, la pareja o los compañeros de trabajo, hablan sobre los candidatos o sus campañas, se descubre que en buena parte de estas interacciones se percibe el partidismo de aquellos con quienes se discute sobre política. Ha de suponerse que el análisis de las interacciones en Twitter mostrará esas relaciones - e identidad- entre grupos sociales. Su importancia radica en que, aun comunicándose en plataformas de internet, esas conversaciones se verifican en el ámbito de lo cotidiano, lo que promueve la descentralización de la esfera pública, ampliándola.

\section{El discurso político en Twitter como práctica social}

Las prácticas sociales son a menudo entendidas como comportamientos recurrentes orientados a un fin específico (Murcia, Jaimes y Gomez, 2016). Tienen un carácter rutinario (Van Dijck, 2012) y articulan la organización social (Ariztía, 2017). Al ser concebidas en función del contexto sociohistórico y cultural en el que se desarrollan, trascienden a la simple ejecución de un comportamiento. Desde hace ya mucho tiempo se tiene conciencia de que el impacto de la tecnología en la vida humana no puede estudiarse sólo a partir de las capacidades de los artilugios tecnológicos en sí, sino que deben realizarse observaciones integrales respecto al uso y al significado que las personas, y particularmente los grupos sociales, dan a la tecnología (Suchman, Blomberg, Orr, y Trigg, 1999). 
Particularmente, el uso que se hace de Twitter puede concebirse como una práctica social mediada, en tanto la herramienta es accesoria a un grupo de actividades que pueden realizarse sin ella: comunicarse, discutir (Van Dijck, 2012). No obstante, las capacidades y características de esta herramienta tecnológica influyen definitivamente en las configuraciones del discurso que es mediado. En el caso de Twitter los llamados hashtags, menciones, retuits y likes son estructuras lingüísticas (Gillen y Merchant, 2013). Cada uno de ellos tiene una intencionalidad que pretende, sobre todo, distinguir a las comunidades digitales. Rosana Reguillo (2018) dice sobre los hashtags:

Los hashtags (etiquetas) son una serie de caracteres simples o compuestos que van precedidos por la tecla numeral, también llamada «gato», \# (...) el hashtag en Twitter opera como una especie de clúster o racimo de nodos o lugares de enunciación articulados por él o los términos de referencia, en el que los usuarios del hashtag colaboran y se encuentran, discrepan, y disputan sentidos o atacan, descalifican, contra-argumentan o amenazan. (Reguillo, 2018, p.17).

Como en toda interacción humana tanto el hashtag como el retuit, trastocan el ámbito cognitivo (texto) y los rituales informativos (contexto) otorgando otro sentido al relato comunicativo. Como ejemplo de lo anterior, Reguillo (2018, p.117) distingue que "la mezcla de etnografía situada, etnografía en línea, y posteriormente el análisis de grandes cantidades de datos llevaron a formular la hipótesis de que el hashtag opera fundamentalmente como un articulador de subjetividades políticas".

Justamente es la discusión política uno de los elementos distintivos que dan propósito a la comunicación a través de Twitter como práctica social mediada. Van Dijck (2012) destaca entre los usos notables de la plataforma su utilidad en campañas, en protestas políticas, en la expresión de desacuerdos, y en la recolección sistemática de opiniones. Gillen y Merchant (2013), identifican además el activismo y el periodismo ciudadano como usos a los que podríamos conceder cierto valor político. Todas estas aplicaciones además denotan un propósito que no puede alcanzarse si no es a partir de la acción comunitaria, de una voluntad de organización.

Existe, asimismo, una discusión respecto al propósito de las prácticas sociales mediadas por Twitter en función de los sujetos y organizaciones que utilizan esta herramienta en el contexto político. Está la postura de que el uso de Twitter se relaciona con una mayor sofisticación política por parte de los ciudadanos que lo utilizan (Muñiz, Echeverría, Rodríguez-Estrada, Díaz-Jiménez, 2016), y se piensa en esta herramienta como facilitadora del intercambio democrático entre ciudadanía e instituciones, puesto que su popularización contribuyó a romper el monopolio informativo y comunicativo de los medios tradicionales (Ruiz, 2015). En un sentido opuesto, se han observado prácticas destinadas a manipular la opinión pública que ponen en evidencia intentos de las élites por controlar la discusión en las redes.

Se ha documentado que la discusión en redes sociales durante los procesos electorales aumenta la participación de la ciudadanía en los comicios, especialmente entre los jóvenes (Aldrich, Gibson, Cantijoch, y Konitzer, 2016; Boulianne, 2015; Effing, Hillegersberg, y Huibers, 2016; Yamamoto, Kushin, y Dalisay, 2015). No obstante, se infiere que el efecto persuasorio del uso de redes sobre la intención de voto del ciudadano debe ser mínimo, debido a que los electores realizan un consumo selectivo de información afín a sus preferencias políticas en medios tradicionales, digitales, y redes sociales (Flaxman, Goel y Rao, 2016). Asimismo, la estructuración de redes a partir de contactos afines ideológicamente propicia la formación de cámaras de eco, en cuyo interior las interacciones sirven sólo para reafirmar la identidad política de los participantes (Barberá, Jost, Nagler, Tucker, y Bonneau, 2015).

Pese a que el efecto es pequeño, el uso estratégico de las redes puede definir el resultado de una elección de forma marginal; no sólo por su capacidad movilizadora o persuasoria, sino también porque se pueden establecer contactos directos con el electorado reduciendo los costos de la publi- 
cidad en medios tradicionales (Francia, 2018). La prensa tradicional sigue teniendo presencia en las discusiones electorales mexicanas en redes sociales, puesto que la ciudadanía utiliza las publicaciones de la prensa para dar soporte a sus opiniones políticas (Hernández, 2019). Pese a la capacidad de las redes para desarrollar interacciones horizontales, se observa que, en los procesos electorales mexicanos, las cuentas de candidatas y candidatos son utilizadas para trasmitir información de forma unidireccional, replicando la lógica de los medios masivos, evidenciando una ausencia de compromiso político con la ciudadanía (Muñiz, Dader, Téllez, y Salazar, 2016).

Justamente porque las redes sociales eluden la mediación de los medios tradicionales, así como las regulaciones legales en términos de propaganda política, las publicaciones en estas plataformas no están sujetos a criterios editoriales, por lo que sus contenidos son más propensos a la agresividad y a la divulgación de noticias falsas (Corona, 2018; Glowacki et al., 2018). Otras prácticas disfuncionales incluyen la generación de contenido y su divulgación de forma inorgánica, a través de la operación de trolls, bots y cyborgs. Este conjunto de prácticas malintencionadas, ha sido documentado en elecciones mexicanas presidenciales desde 2012, involucrando las campañas de los principales contendientes (Magallón, 2019; Moo-Mena, Robles-Sandoval, González-Magaña, y Rodríguez-Adame; 2019).

\section{Metodología.}

La construcción metodológica del análisis de los tuits siguió los siguientes pasos:

1) Se recolectaron $1,272,280$ tuits durante los tres meses en que transcurrió la campaña, del 30 de marzo al 27 de junio del 2018.

2) El primer filtro consistió en seleccionar la mención de alguno de los tres candidatos principales: Andrés Manuel López Obrador, de la alianza MORENA-PES-MC; José Antonio Meade, de la alianza PRI-VERDE-PANAL, y Ricardo Anaya de la alianza PAN-PRD. Esta recolección se realizó utilizando el paquete $r$ tweet, en el entorno de programación R (Kearney, 2016) ${ }^{2}$.

3) Los tuits obtenidos fueron procesados excluyendo los retuits, y sólo se analizaron aquellos tuits originales que generaron más de diez reacciones de "me gusta" (likes). Estos filtros resultaron en un corpus o base de datos final de 4664 tuits.

\subsection{La propuesta del Libro de Código para analizar Twitter.}

El Libro de Código es el instrumento que con técnicas de análisis de contenido permite el registro de la información para aplicar en distintos sistemas comunicativos: noticias impresas en periódicos, textos de grandes discursos, o publicaciones en Twitter. Para ello el investigador tiene que reconocer las características tanto estructurales como cognitivas del discurso que analiza.

Las preguntas de investigación que orientaron el trabajo de campo surgieron de las reflexiones teóricas anteriores, con el objetivo de evaluar el comportamiento de las comunidades digitales en Twitter durante las elecciones 2018 en México:

1.- ¿Cuáles fueron las características estructurales del lenguaje utilizado en Twitter durante la campaña electoral?

2.- ¿Quiénes son los emisores de información en la interacción durante la campaña y qué temas abordaron?

\footnotetext{
2 Kearney, M.W.: rtweet. [Package rtweet version 0.2.4] (2016). https://CRAN.R-project.org/package=rtweet
} 
3.- ¿Qué sentimientos expresan las comunidades del twitter hacia cuál candidato?

4.- ¿Los ciudadanos digitales vinculan ("linkean”) información que emiten los medios en la web?

El libro tuvo por Unidad de Análisis los tuits cuyo referente mencionara a alguno de estos candidatos: José Antonio Meade (PRI-Verde-Alianza), Ricardo Anaya (PAN- PRD), o Andrés Manuel López Obrador (MORENA, PES, Movimiento), y/o sus equivalentes (AMLO, Meade, etc.).

Las variables de análisis fueron las características estructurales del lenguaje de Twitter: tuits, retuits, las etiquetas o hashtags y los likes. La dimensión cognitiva del mensaje, que representa la individual interpretación de la realidad, se estudió a través de los sentimientos que resultaron de esas interacciones.

Se construyó una base de datos que analizó, mención por mención, el sentido de la interacción acerca del candidato. Dicho corpus se compone de las variables que se presentan en la tabla 1.

Tabla 1. Variables que componen la base de datos

\begin{tabular}{|c|c|c|}
\hline Variable & \multicolumn{2}{|l|}{ Descripción } \\
\hline Fecha & \multicolumn{2}{|c|}{ Fecha de emisión de tuit } \\
\hline Usuario & \multicolumn{2}{|c|}{ Nombre de la cuenta de usuario } \\
\hline Emisor & \multicolumn{2}{|c|}{$\begin{array}{l}\text { Ciudadanos comunes: Cuando el nombre del usuario no permite una identificación en las } \\
\text { categorías posteriores. } \\
\text { Medios de Comunicación: Cuentas oficiales de los medios de comunicación. } \\
\text { Periodistas: Cuentas personales de los periodistas, sin relación con el medio) } \\
\text { Sociedad Civil: Empresarios, sindicatos, inversionistas, agrupaciones empresariales, de- } \\
\text { portistas, actores, cantantes, líderes religiosos, que se logran identificar. } \\
\text { Partidos políticos: los que se identificaron. }\end{array}$} \\
\hline Seguidores & \multicolumn{2}{|c|}{ Número de seguidores que tiene la cuenta } \\
\hline Localización & \multicolumn{2}{|c|}{ Lugar de emisión del tuit } \\
\hline Texto & \multicolumn{2}{|l|}{ Texto del tuit } \\
\hline Me gusta & \multicolumn{2}{|c|}{ Número de reacciones "Me gusta" del tuit } \\
\hline Retuits & \multicolumn{2}{|c|}{ Número de retuits } \\
\hline Hashtags & \multicolumn{2}{|c|}{ Cuando el mensaje tenía al menos una etiqueta } \\
\hline Mención & \multicolumn{2}{|c|}{ Si el tuit mencionó a otro usuario } \\
\hline Actor & \multicolumn{2}{|c|}{ Candidato a la presidencia mencionado en el tuit } \\
\hline Sentimiento & \multicolumn{2}{|c|}{ Valoración, positivo, negativo y neutral de las expresiones del texto en el tuit } \\
\hline \multirow[b]{2}{*}{ Asunto } & Argumento & Opiniones acerca de los candidatos: personalidad y/o campaña \\
\hline & $\begin{array}{l}\text { Tema: Políticas } \\
\text { públicas }\end{array}$ & $\begin{array}{l}\text { Corrupción, seguridad pública, transparencia y acceso a la información, } \\
\text { economía, infraestructura, educación. }\end{array}$ \\
\hline Links & \multicolumn{2}{|c|}{ Cuando el tuit tuviera un link dentro del texto } \\
\hline Contenido del link & \multicolumn{2}{|c|}{ Notas periodísticas, fotos en redes sociales } \\
\hline
\end{tabular}

\section{Resultados}

Este trabajo presenta el análisis de 4664 tuits publicados durante los tres meses que transcurrió la campaña electoral 2018 en México. Se presentan las gráficas de los tuits analizados por las variables representativas de acuerdo a las preguntas de investigación que orientaron el libro de códigos y el objetivo general de esta investigación. 
Figura 1. Total de tuits analizados por candidato.

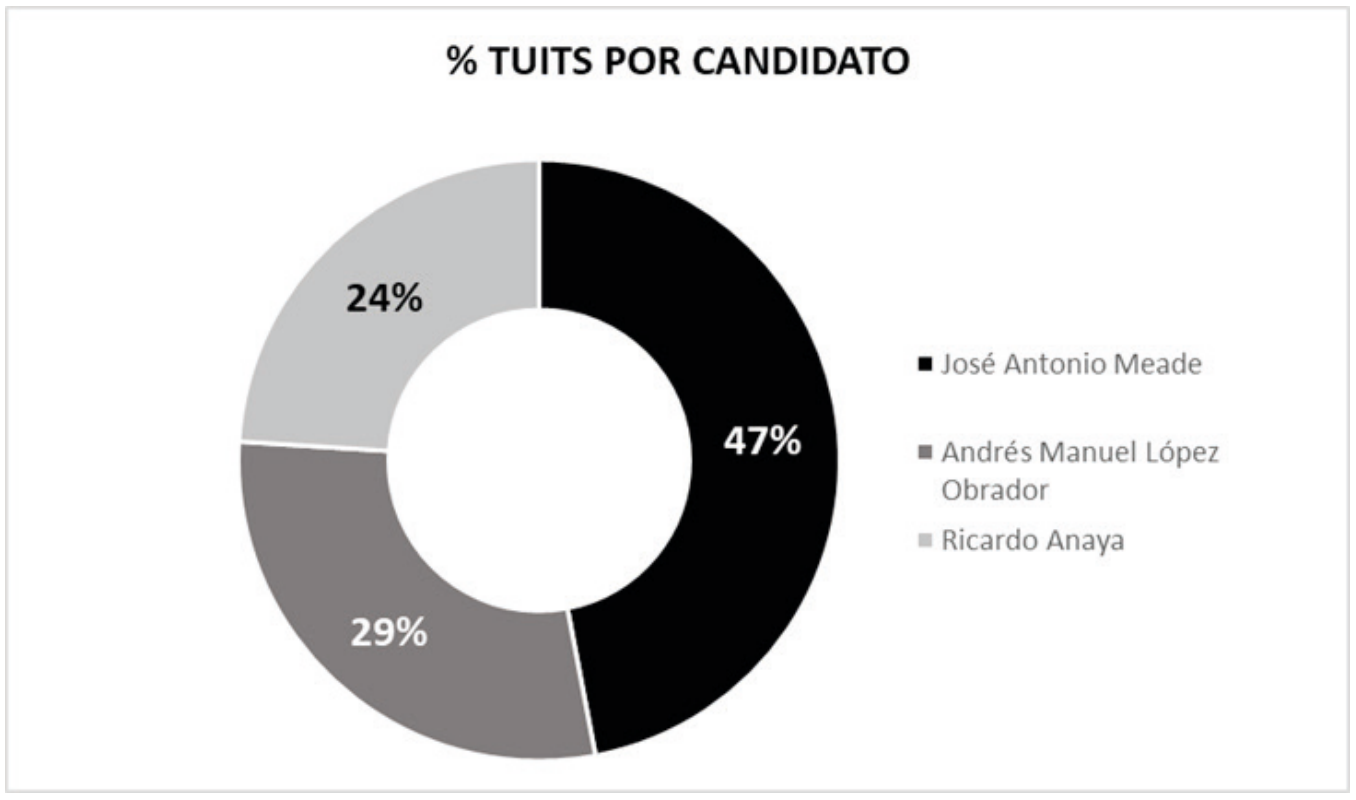

Fuente: Elaboración propia.

La figura 1 muestra que el $47 \%$ de los tuits emitidos por los usuarios hablaron sobre el candidato José Antonio Meade, teniendo la mayor presencia en los mensajes analizados.

Figura 2. Número de tuits por candidato y sentimiento (Positivo, Negativo, Neutral)

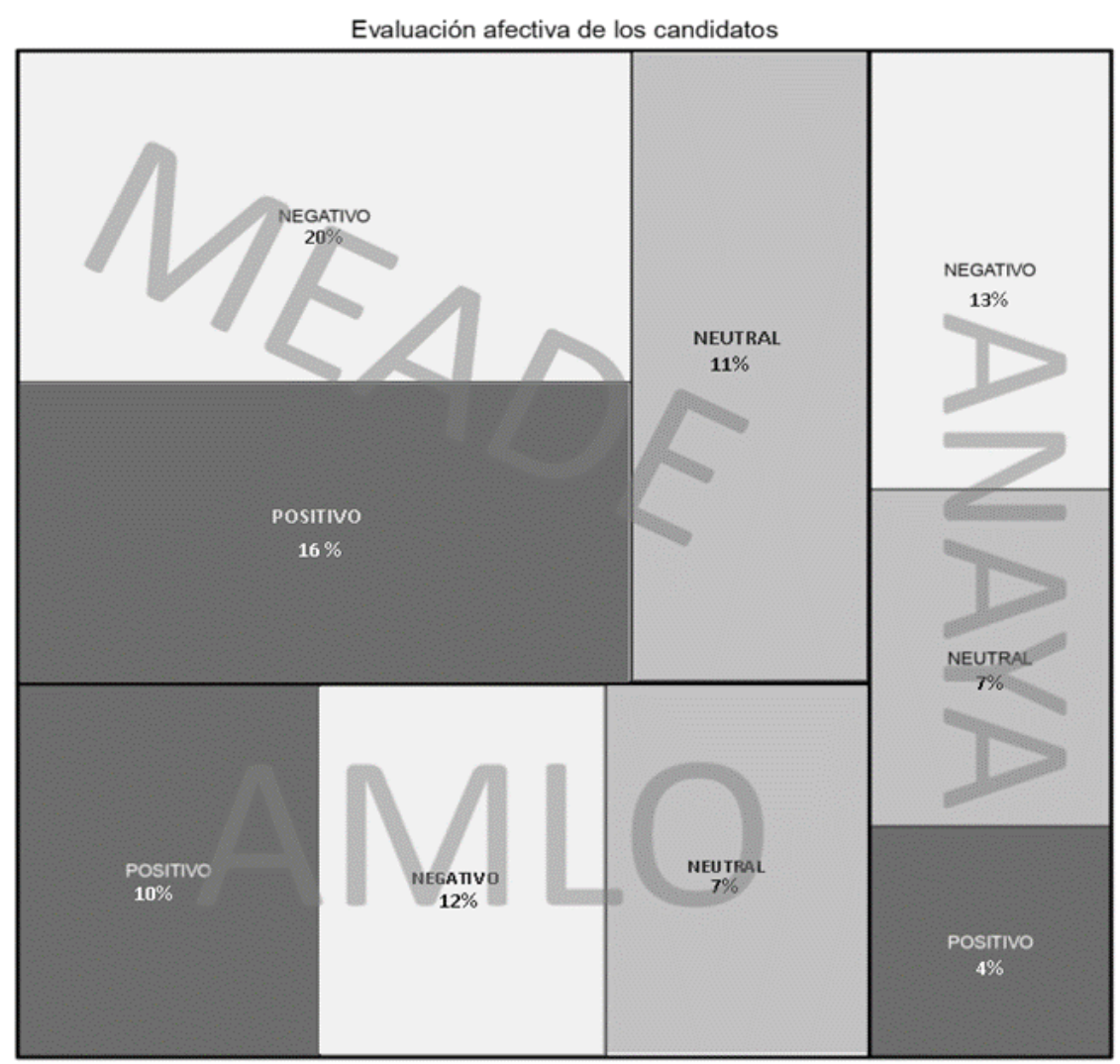

SENTIMIENTO

$\square$ NEGATIVO

$\square$ NEUTRAL

$\square$ POSITIVO

Fuente: Elaboración propia. 
En la figura 2 se observa la presencia mayoritaria de las comunidades digitales que expusieron sus opiniones en contra de los candidatos y/o sus campañas a través de tuits con sentimiento negativo (47\%), José Antonio Meade obtuvo el mayor porcentaje negativo (20\%), seguido de Ricardo Anaya (13\%) y Andrés Manuel López Obrador (12\%). Con respecto a los tuits de apoyo, Meade tuvo el $16 \%$ de tuits positivos.

Figura 3. Número de tuits por candidato, localización y sentimiento

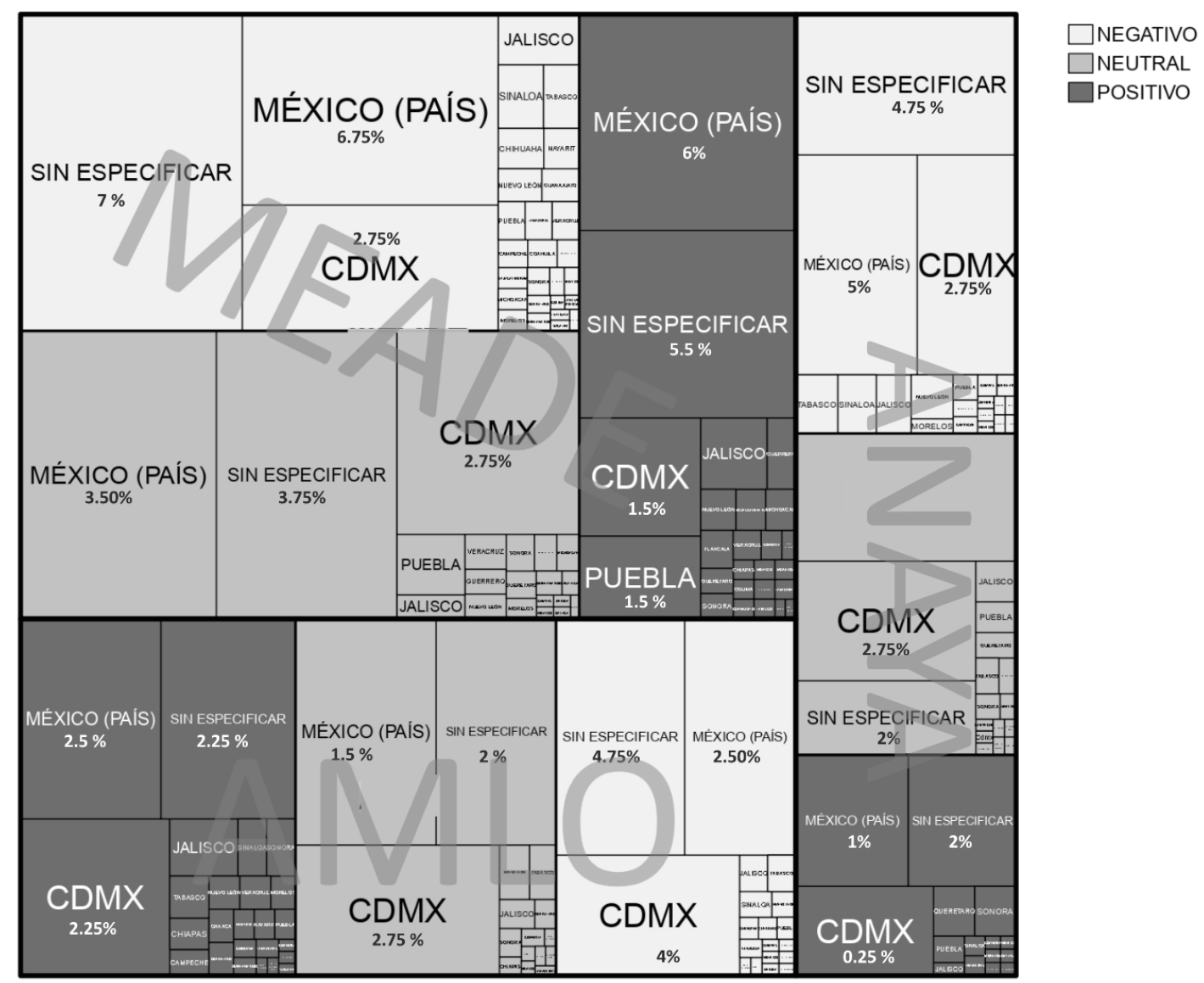

Fuente: Elaboración propia.

La figura 3 muestra las interacciones de los usuarios de Twitter sobre las campañas y sus candidatos, destacaron de la comunidad de usuarios provenientes de la Ciudad de México donde se emitió el $21.25 \%$ de los tuits y quienes reflejaron su negativa hacia Andrés Manuel López Obrador con un 4\%. También se observa la participación de los estados de Jalisco (2.50\%) y Puebla (2.25\%) siendo Andrés Manuel $(1.5 \%)$ y Meade (1.5\%) respectivamente, los candidatos apoyados. Se reflejó una mínima participación dentro de la categoría "otros estados" (7\%) conformada por nueve estados con menos del 1\%. La localización denominada "México" (país) corresponde a los tuits sin identificar su lugar de procedencia. 
Figura 4. Tuits por emisor, candidato y sentimiento (usuarios agrupados por categorías).

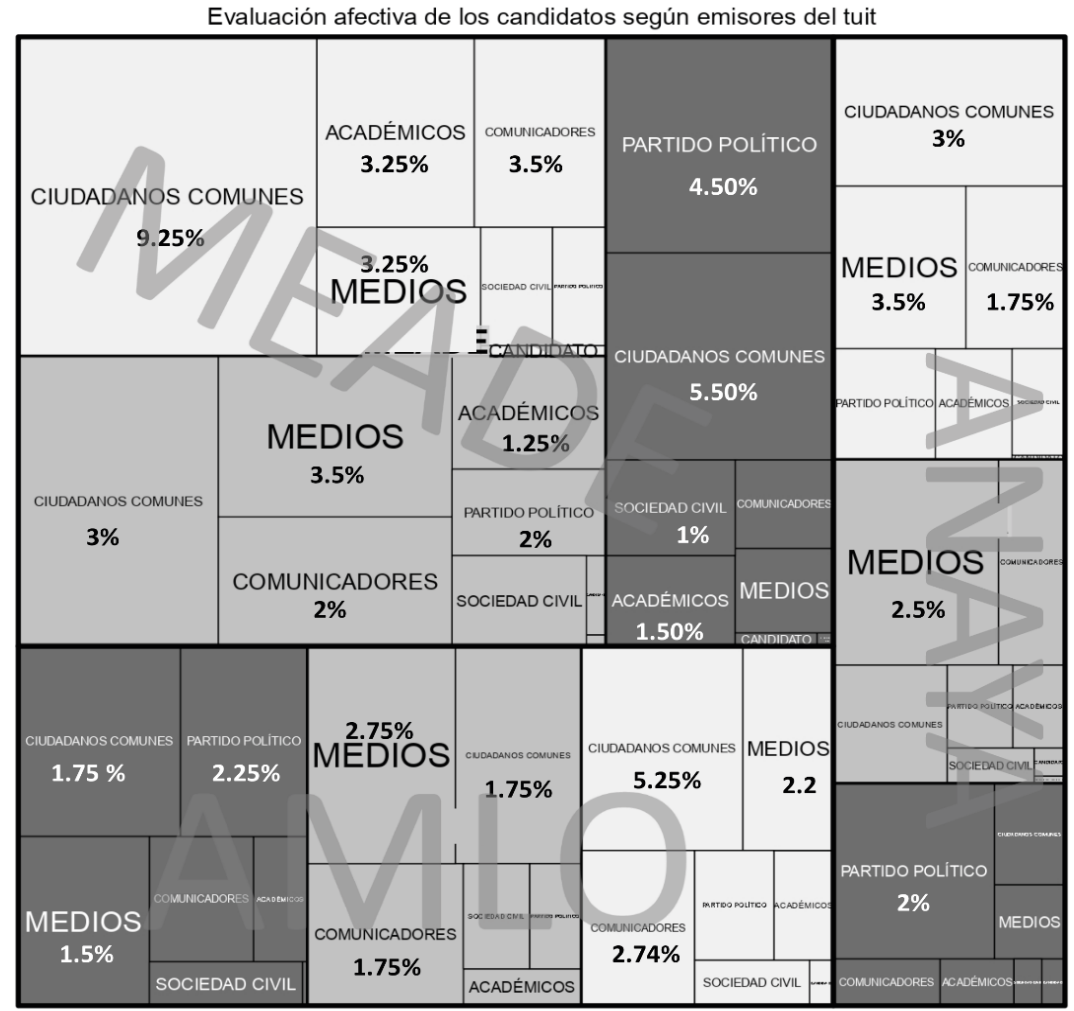

SENTIMIENTO

$\square$ NEGATIVO

$\square$ NEUTRAL

$\square$ POSITIVO

Fuente: Elaboración propia.

Figura 5. Asunto del tuit por candidato y sentimiento

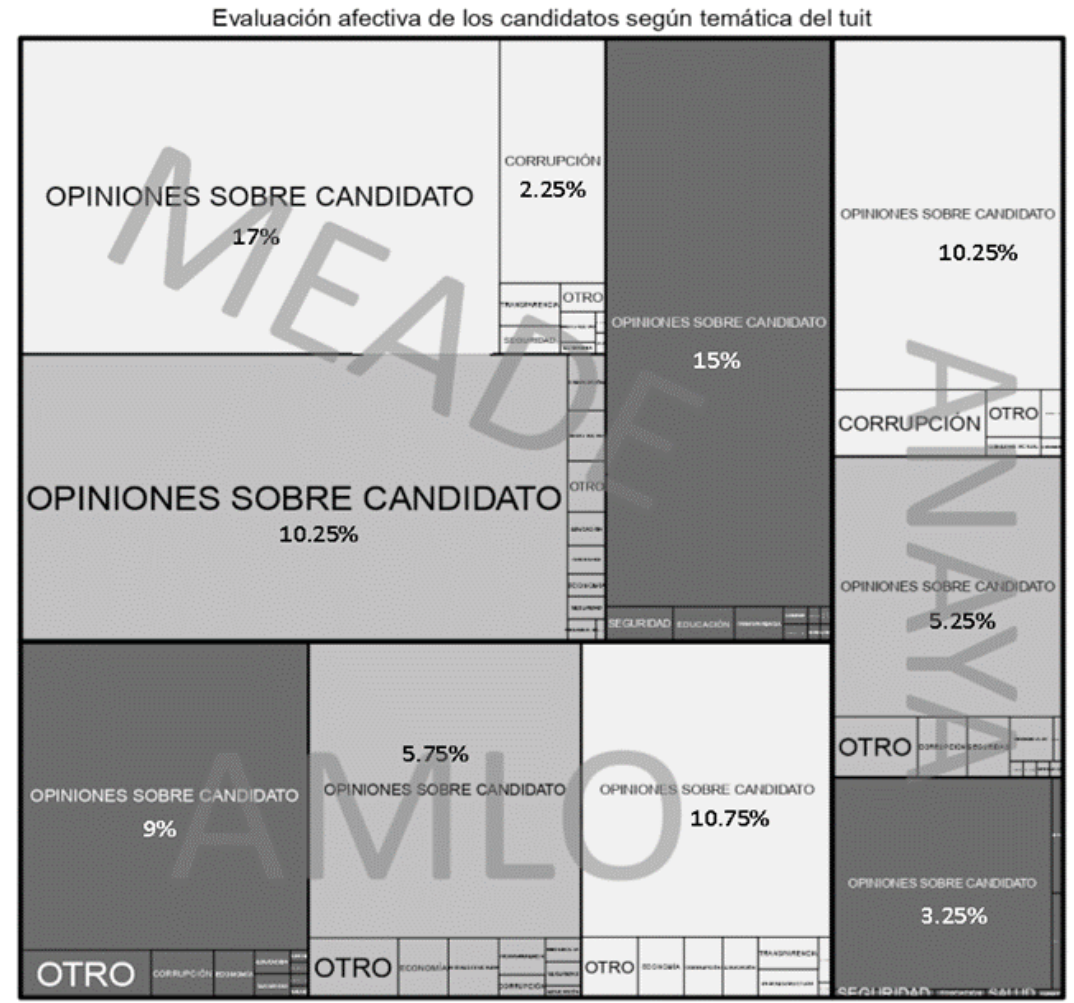


Como se muestra en la figura 4, los usuariosde Twitter catalogados como "ciudadanos comunes" (31\%) fueron la comunidad digital con más tuits emitidos sobre las Elecciones 2018 y quienes más tuitearon en negativo sobre los tres candidatos, de los cuáles José Antonio Meade obtuvo el mayor porcentaje $(9.25 \%)$. Los "periodistas" reflejan un comportamiento similar a los "ciudadanos comunes", mientras que los "medios de comunicación" mantienen su lado neutral; en conjunto reúnen el $40 \%$ del total de los tuits emitidos sobre las campañas políticas en Twitter. El apoyo hacia los candidatos provino de las cuentas de sus "partidos políticos".

Respecto a los asuntos que se trataron en los tuits, en la figura 5 se observa que la comunidad digital interactuó en su mayoría sobre la personalidad de los candidatos y/o su campaña (87\%), donde predomina el sentimiento negativo en los tres candidatos. Del total de tuits, el 13\% trataron sobre políticas públicas de las cuales el 5\% destaca el asunto de la "Corrupción; en ese tema se presentaron más negativos respecto a José Antonio Meade (2.25\%). Quien resulta con menos negativos en el mismo tema es Andrés Manuel López Obrador (0.50\%).

Tabla 2. Recursos multimedia del link que compartieron los emisores

\begin{tabular}{|c|c|c|c|c|c|c|c|c|}
\hline $\begin{array}{l}\text { Contenido del } \\
\text { link }\end{array}$ & $\begin{array}{c}\text { Medios de } \\
\text { Comunicación }\end{array}$ & Periodistas & $\begin{array}{l}\text { Partido } \\
\text { Político }\end{array}$ & $\begin{array}{c}\text { Ciudadanos } \\
\text { Comunes }\end{array}$ & $\begin{array}{l}\text { Académicos/ } \\
\text { Especialistas }\end{array}$ & $\begin{array}{c}\text { Sociedad } \\
\text { Civil }\end{array}$ & Otros & $\begin{array}{c}\text { TOTAL } \\
\%\end{array}$ \\
\hline $\begin{array}{l}\text { Noticias } \\
\text { en internet } \\
\text { (portales } \\
\text { web, videos, } \\
\text { transmisiones } \\
\text { en vivo) }\end{array}$ & $12.7 \%$ & $4.1 \%$ & $1.3 \%$ & $1.3 \%$ & $1.0 \%$ & $0.8 \%$ & $0.0 \%$ & $21.2 \%$ \\
\hline $\begin{array}{l}\text { Fotos en } \\
\text { redes sociales }\end{array}$ & $1.0 \%$ & $1.6 \%$ & $2.3 \%$ & $2.7 \%$ & $0.8 \%$ & $0.4 \%$ & $0.2 \%$ & $9.0 \%$ \\
\hline No aplica & $6.1 \%$ & $9.2 \%$ & $11.4 \%$ & $30.2 \%$ & $7.3 \%$ & $4.9 \%$ & $0.6 \%$ & $69.8 \%$ \\
\hline TOTAL & $19.8 \%$ & $14.9 \%$ & $15.0 \%$ & $34.2 \%$ & $9.2 \%$ & $6.1 \%$ & $0.8 \%$ & $100.0 \%$ \\
\hline
\end{tabular}

Fuente: Elaboración propia

En la tabla 2 se pueden ver los recursos multimedia del link que compartieron los emisores, donde el $30.2 \%$ de los tuits recurrieron a un link en el cuerpo de sus mensajes como complemento a sus textos. En cuanto a las notas compartidas desde un portal web, vemos que sólo el $21.2 \%$ de los tuits analizados se apoyaron en este tipo de información. Los que más recurrieron a ellas son los propios medios de comunicación (12.7\%) y los que menos la sociedad civil $(0.8 \%)$, académicos o especialistas (1\%) y los ciudadanos comunes (1.3\%). El 69.8\% de la comunidad digital no vincula la información de otros medios en sus tuits, sino que sólo expresan sus sentimientos sobre las campañas y sus candidatos.

La figura 6 presenta tres gráficos que muestran los hashtags utilizados por los usuarios al emitir sus textos hacia uno de los candidatos. En los tuits que mencionaron a Andrés Manuel López Obrador predominaron los hashtags positivos como \#AMLO o \#JuntosHaremosHistoria, sin embargo, también fueron ocupados en tuits con carga negativa y neutra. Por su parte, los tuits sobre Ricardo Anaya se complementaron en su mayoría con hashtags positivos como \#DeFrenteAlFuturo y \#MujeresConAnaya. Los tuits que mencionaron al candidato José Antonio Meade emplearon un mayor número de hashtags para favorecerlo, siendo \#MeadePresidente el más apoyado. 
Andrade del Cid, P., Flores González, R. y Pablo Contreras, M.

Figura 6. Hashtags utilizados por candidato y sentimiento
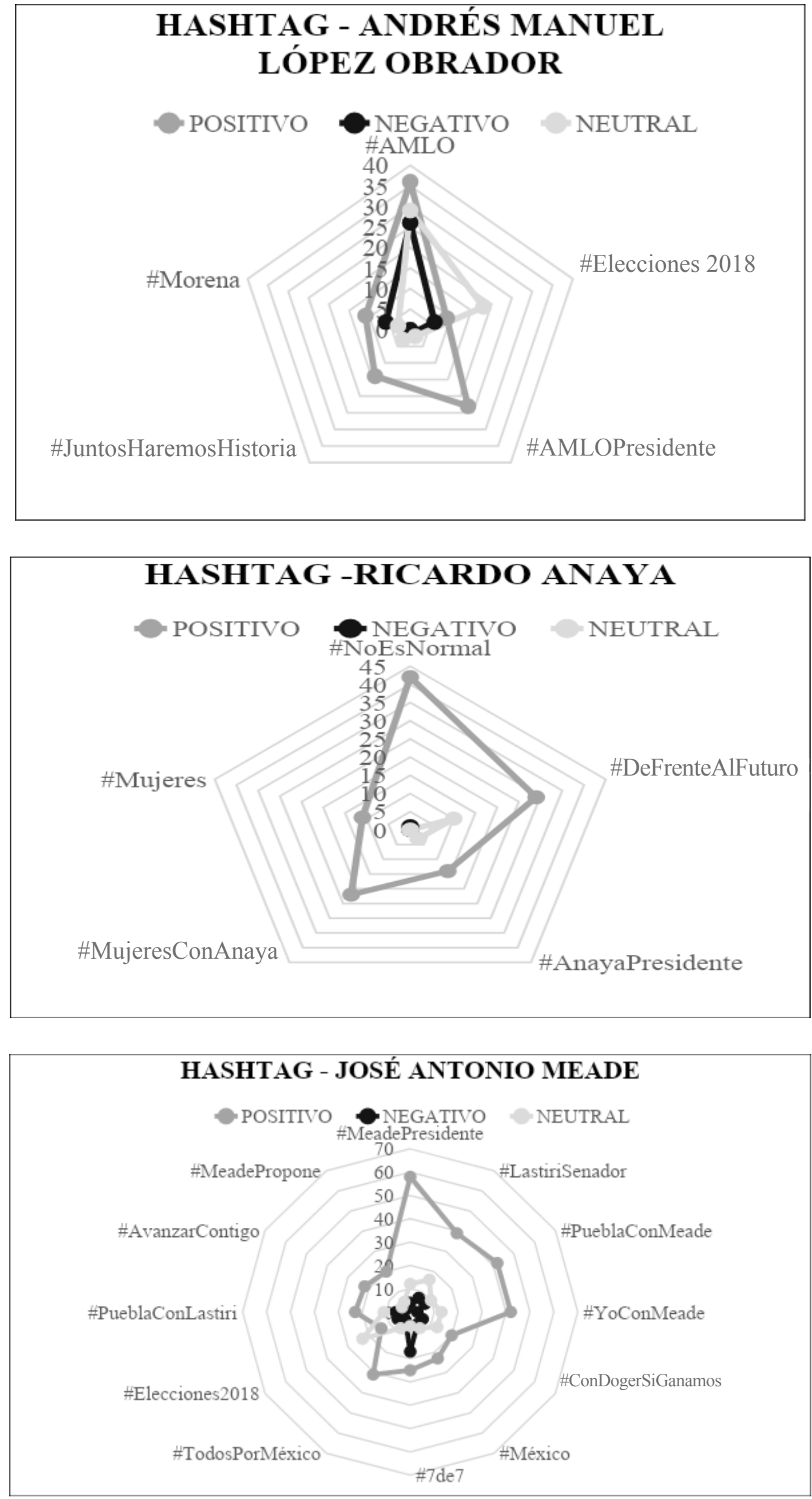

Fuente: Elaboración propia 
Tabla 3. Tuits más compartidos (número de retuits)

\begin{tabular}{|c|c|c|c|c|}
\hline \multicolumn{5}{|c|}{ Tuits con mayor número de retuits } \\
\hline Usuario & Tuit & $\begin{array}{c}\text { Me } \\
\text { gusta }\end{array}$ & Retuit & Sentimiento \\
\hline \multicolumn{5}{|c|}{ Tuits más retuiteados que mencionan a Andrés Manuel López Obrador, AMLO, Andrés. } \\
\hline @ JoseAMeadeK & $\begin{array}{l}\text { No, @lopezobrador_, no es una provocación ni se trata } \\
\text { de que te enojes. Y tú, @RicardoAnayaC, podrás correr } \\
\text { mas no huir. El que nada debe, nada teme. \#NoLeSa- } \\
\text { quen. Reitero el reto de transparentar públicamente nues- } \\
\text { tro patrimonio personal y familiar. }\end{array}$ & 3096 & 1884 & Negativo \\
\hline @JoseAMeadeK & $\begin{array}{l}\text { Andrés: deja de ocuparte de mí y comienza a preocupar- } \\
\text { te por ti. Perderás otra vez. Quisieras quedarte sin rival, } \\
\text { pero lamento decirte que ahí viene tu tercer strike }\end{array}$ & 2665 & 1141 & Negativo \\
\hline @JoseAMeadeK & $\begin{array}{l}\text { No sé qué esté peor: la hipocresía de repudiar los vuelos } \\
\text { privados, pero echar mano de ellos; o mentir al afirmar } \\
\text { que se trató de un taxi aéreo, siendo realmente una aero- } \\
\text { nave de transporte privado. Es AMLO. No cambia. }\end{array}$ & 1640 & 845 & Negativo \\
\hline \multicolumn{5}{|c|}{ Tuits más retuiteados que mencionan a José Antonio Meade, Meade. } \\
\hline @brozoxmiswebs & $\begin{array}{l}\text { Meade avaló en febrero de } 2010 \text { el contrato entre Pemex } \\
\text { y Braskem, filial de Odebrecht, para que en conjunto con } \\
\text { la empresa mexicana Idesa pusieran en marcha la planta } \\
\text { Etileno XXI; en dicho contrato Pemex le vendió su alma al } \\
\text { diablo./“Sin Desperdicio” }\end{array}$ & 1185 & 1435 & Negativo \\
\hline @JoseAMeadeK & $\begin{array}{l}\text { Conducirse con prejuicios, descalificaciones personales y } \\
\text { atacar a quienes generan empleos e inversiones por Mé- } \\
\text { xico es un despropósito y una amenaza. Yo, en cambio, } \\
\text { ofrezco brindar seguridad jurídica y aumentar la competiti- } \\
\text { vidad del país. }\end{array}$ & 1574 & 848 & Positivo \\
\hline @JoseAMeadeK & $\begin{array}{l}\text { Hay muros que nos distancian y otros que salvan vidas. } \\
\text { En unas horas haré un importante anuncio desde la fron- } \\
\text { tera norte. Estén pendientes. }\end{array}$ & 1226 & 689 & Positivo \\
\hline \multicolumn{5}{|c|}{ Tuits más retuiteados que mencionan a Ricardo Anaya } \\
\hline @RicardoAnayaC & $\begin{array}{l}\text { Estoy convencido de que debemos lograr un cambio } \\
\text { profundo en \#México. Nuestro país lo tiene todo, pero } \\
\text { nos han faltado buenos gobiernos. Voy a ser Presidente } \\
\text { de México, para que juntos logremos ese cambio que } \\
\text { nuestro país necesita. En @EnfoqueNoticias con @Ma- } \\
\text { rioCNN. }\end{array}$ & 319 & 921 & Positivo \\
\hline @RicardoAnayaC & $\begin{array}{l}\text { Invito a toda la gente a que el } 1 \text { de julio salgamos a vo- } \\
\text { tar por las candidatas y los candidatos de la coalición } \\
\text { \#PorMéxicoAlFrente, para tener funcionarios y gobiernos } \\
\text { honestos y de resultados. En @EnfoqueNoticias con @ } \\
\text { MarioCNN. }\end{array}$ & 295 & 692 & Positivo \\
\hline @alvaro delgado & $\begin{array}{l}\text { Reúne Carlos Salinas a amigos y a los tres poderes: } \\
\text { Peña y su séquito; Fernández de Cevallos, asesor de } \\
\text { @RicardoAnayaC; la representante de @ JoseAMea- } \\
\text { deK, Vanessa Rubio; el cacique petrolero Romero Des- } \\
\text { champs... }\end{array}$ & 161 & 407 & Negativo \\
\hline
\end{tabular}

\section{Fuente: Elaboración propia}

En la tabla 3 se presentan los tuits con más likes y más retuits, fueron los que emitió José Antonio Meade de la coalición PRI-Verde-Nueva Alianza contra AMLO de Morena. De los que tratan sobre José Antonio Meade, el más retuiteado tiene tendencia negativa y lo emite un periodista. Los que son emitidos por los propios candidatos tienen tendencia positiva, y los de los periodistas son negativos hacia el candidato que mencionan. 


\section{Conclusiones}

Si la cultura política obedece a reglas normativas cuyo referente evalúa las actitudes, este análisis nos confirma que el comportamiento electoral tuvo "cierta base cultural sobre fenómenos políticos" (Piñuel y Gaitán, 1995); así, se señala a "la corrupción", en particular la del partido que gobernaba (PRI), como el tema más preocupante.

A continuación, los hallazgos más relevantes:

1) La mayoría de los tuits emitidos correspondieron al candidato J. Antonio Meade del PRI, pero la tendencia fue negativa; este comportamiento fue el mismo para Ricardo Anaya y Andrés Manuel López Obrador.

2) Los emisores llamados "usuarios comunes" emiten sus ideas desde la metrópoli, lo que se corresponde a la participación social y las visiones de la democracia desde la capital, conquistada por la izquierda mexicana desde hace más de 20 años. Suponemos que, a consecuencia de esa particular participación, la mayor parte de los tuits son negativos, sumando $46 \%$.

3) Los "ciudadanos comunes" emiten más tuits negativos hacia el candidato del partido en el poder (PRI) José Antonio Meade; aunque también lo hacen "los periodistas" y "especialistas" en el tema. En cambio "los medios de comunicación" se muestran neutrales con los candidatos analizados.

4) A pesar de que los medios contribuyen en sólo con $21.75 \%$ en la discusión en las redes, si lo sumamos a "los periodistas", la suma llega a casi el $40 \%$ de los tuits analizados.

5) En cuanto a los asuntos o temas: el $87 \%$ de los tuits tratan sobre la "personalidad" o "campaña" de los candidatos. Cuando analizamos cada texto del tuit (argumento) vemos que la mayoría de los negativos los obtiene Meade (17\%) pero también los candidatos Ricardo Anaya (10.25\%) y Andrés Manuel López Obrador (10.75\%), sin embargo, este último también obtiene un número considerable de positivos.

6) En ese análisis el tema "corrupción" es el que tiene más comentarios negativos para Meade, candidato del PRI-Verde-Alianza.

7) El análisis de los hashtags más representativos nos muestra que Meade (PRI) los utilizó más y fueron de tendencia positiva. En cambio, los retuits más numerosos de José Antonio Meade fueron de la cuenta de un periodista - @brozoxmiswebs - con tendencia negativa.

8) La comunicación social que ejerció la ciudadanía digital se centralizó en la Ciudad de México (21.25\%) y se distinguió por emitir el $47 \%$ de los mensajes negativos hacia cualquiera de los candidatos

Respecto a los resultados de esas elecciones, el candidato Andrés Manuel López Obrador de MORENA ganó las elecciones 2018 con el $53.19 \%$ del total de los votos. Esta victoria no se explica sin comprender que el país ha vivido una crisis muy aguda en el ámbito político y económico ${ }^{3}$.

\section{Discusión}

\footnotetext{
${ }^{3}$ Javier Esteinoú en la Revista Zócalo de agosto 2018, describe los factores políticos que decantaron el voto hacia AMLO: “... la extendida corrupción, la desbordada inseguridad, la escandalosa impunidad, la enorme desigualdad, la aguda marginación y el creciente desprestigio de la clase política" (p.26).
} 
Este trabajo tuvo por objetivo evaluar la importancia de la comunicación digital durante campañas electorales a través del comportamiento de las comunidades digitales del Twitter, porque la comunicación implica comportamiento y durante las elecciones esa conducta se amplía, dejando rastros o subjetividades políticas en su discurso.

En relación al lenguaje del Twitter, los partidarios de cada candidato ejercieron su "poder" utilizando sus estructuras a través del retuit y el like, mientras que el hashtag es utilizado por los partidos, como una extensión de sus actividades en la búsqueda de identidad. Esta estructura particular - etiqueta o hashtag - puede no tener la fuerza que demuestra en los movimientos sociales, pero su utilización en campaña política busca ser un vínculo con sus correligionarios. Esta tendencia muestra que la identidad política del ciudadano digital se construye menos por ideología política y más por representaciones sociales: la teoría al respecto afirma que son elementos valorativos los que orientan la postura del sujeto frente al objeto representado y que determinan su conducta hacia él (Ibáñez, 1994).

Cuando se elaboró el análisis de las estructuras del lenguaje (hashtags, retuits, likes) y de las dimensiones cognitivas (positivo/negativo/neutral), lo que se buscó fue reconstruir esa subjetividad política, a través de las interacciones que se verifican en esa red social y que implican un comportamiento por el carácter evaluativo y normativo a través de la comunicación. Consideramos que la democracia deliberativa encuentra su lugar en las redes, en dónde se representan los elementos valorativos que identifican a los partidarios de uno u otro, y van construyendo y re-construyendo su identidad; esta actividad permite que se amplíe la esfera pública. Sin embargo, a pesar de esa expansión, este trabajo - construido tanto por procesos informáticos como por técnicas de análisis de contenido-, demuestra que las prácticas sociales hegemónicas de la comunicación y cultura política prevalecen, porque los medios de comunicación y sus periodistas fueron los principales emisores, al sumar entre los dos casi 40\% de los tuits analizados, superando el $31 \%$ de los llamados "ciudadanos comunes". Lo cual manifiesta la reproducción de la opinión pública como pensamiento dominante en la red social Twitter.

\section{Bibliografía}

Abascal, R. (2015). Comunicación política en 140 caracteres: el caso \# Ayotzinapa, en Razón y Palabra. 19 (92), 1-30.

Aldrich, J., Gibson, R., Cantijoch, M., y Konitzer, T. (2016). Getting out the vote in the social media era: Are digital tools changing the extent, nature and impact of party contacting in elections? Party Politics, 22(2), 165-178. https://doi.org/10.1177/1354068815605304

Andrade, P y Castro, C. (2014). El discurso de tres candidatos a la presidencia de México en la prensa impresa. Estrategias de las herramientas de análisis estadístico de datos textuales. Revista Legislativa de Estudios Sociales y de Opinión Pública, 7(4).

Andrade, P. (2007). Relatos del Periodismo Veracruzano. El presente en la noticia. IEV.

Ariztía, T. (2017). La teoría de las prácticas sociales: particularidades, posibilidades y límites. Cinta de Moebio, 59, 221-234.

Azurmendi, A., Muñoz Saldaña, M., \& Ortega Mohedano, F. (2018). Metodologías Avanzadas de Investigación en Comunicación y Ciencias Sociales, la revolución de los instrumentos y los métodos, Qualtrics, Big Data, Web Data., en Tendencias Metodológicas en la investigación académica de la comunicación. COMUNICACIÓN SOCIAL. Ediciones y publicaciones.

Barberá, P., Jost, J., Nagler, J., Tucker, J., y Bonneau, R. (2015). Tweeting From Left to Right: Is Online Political Communication More Than an Echo Chamber? Psychological Science, 26(10), 
1531-1542. https://doi.org/10.1177/0956797615594620

Boulianne, S. (2015). Social media use and participation: a meta-analysis of current research. Information, Communication and Society, 18(5), 524-538. https://doi. org/10.1080/1369118X.2015.1008542

Castells, M. (1999). La Era de La Información. Siglo XXI Editores.

Corona, A. (2018). Mecanismos de otrificación entre la oposición política en Twitter durante las elecciones estatales de 2017 en México. adComunica, 16, 45-70.

Effing, R., Hillegersberg, J., y Huibers, T. (2016). Social Media Indicator and Local Elections in the Netherlands: Towards a Framework for Evaluating the Influence of Twitter, YouTube, and Facebook. En Sobaci, M. (ed). Social Media and Local Governments, 281-298. Springer-Cham https://doi.org/10.1007/978-3-319-17722-9_15

Esteinoú J. (agosto 2018) Porqué ganó López Obrador en 2018?: los factores políticos. Zócalo, (222), 25-27.

Flaxman, S., Goel, S. y Rao, J. (2016). Filter bubbles, echo chambers, and online news consumption. Public Opinion Quarterly, 80(S1), 298-320. https://doi.org/10.1093/poq/nfw006

Francia, P. L. (2018). Free media and Twitter in the 2016 presidential election: the unconventional campaign of Donald Trump. Social Science Computer Review, 36(4), 440-455. https://doi. org/10.1177/0894439317730302

Gillen, J. y Merchant, G. (2013). Twitter as a dialogic and linguistic practice. Language Sciences, $35,47-58$.

Glowacki, M., Narayanan, V., Maynard, S., Hirsch, G., Kollanyi, B., Neudert, L., Howard, P., Lederer, T., y Barash, V. (2018). News and Political Information Consumption in Mexico: Mapping the 2018 Mexican Presidential Election on Twitter and Facebook (Vol. 29). COMPROP DATA MEMO 2018.2/JUNE. http://comprop.oii.ox.ac.uk/wp-content/uploads/sites/93/2018/06/ Mexico2018.pdf

Hernández, M. (2019). La presencia de AMLO en twitter en la precampaña de las elecciones federales de 2018 en México. Tesis de especialización. Xalapa, MX: Universidad Veracruzana.

Hardt, M., y Negri, A. (2004). Multitud: guerra y democracia en la era del Imperio. Editorial Debate.

Ibáñez, J. (1994). El regreso del sujeto la investigación social de segundo orden. Siglo XXI Editores.

Kearney, M. W. (2016). Rtweet : Collecting Twitter Data (Versión 0.1.7) [Software]. https://cran.rproject.org/package $=$ rtweet

Kellner, D. (2000). Habermas, the public sphere, and democracy: A critical intervention. En Hahn, L. (ed.), Perspectives on Habermas, Pp. 259-288. Open Court Press.

Magallón, R. (2019). Verificado México 2018. Desinformación y fact-checking en campaña electoral. Revista de Comunicación, 18(1), 234-258. https://doi.org/10.26441/RC18.1-2019-A12

Maldonado G. (2012). Intermediarios políticos y comportamiento electoral en México: dime con quién hablas y te diré por quién votas. El comportamiento electoral mexicano en las elecciones 2012., Meixueiro y Moreno (coords.). CESOP/ ITAM 2014. 
Menesses M. (2015) en Winocur, R. y Sánchez, J. Redes sociodigitales en México. México: Fondo de Cultura Económica y Consejo Nacional para la Cultura y las Artes (Biblioteca Mexicana). 40-6. México: FCE y CONACULTA.

Molina, J. L. (2011). Los cambios culturales provocados por el software social. Lychnos. http:// www.fgcsic.es/lychnos/es_es/articulos/los_cambios_culturales_provocados_por_el_software_social

Moo-Mena, F., Robles-Sandoval, S., González-Magaña, K., y Rodríguez-Adame, O. (2019). Towards bots detection by analyzing the behavior of user data on twitter. International Journal of Computer Science Issues (IJCSI), 16(1), 21-29. http://dx.doi.org/10.5281/zenodo.2588241

Muñiz, C., Dader, J. L., Téllez, N. M., y Salazar, A. (2016). ¿Están los políticos políticamente comprometidos?: Análisis del compromiso político 2.0 desarrollado por los candidatos a través de Facebook. Cuadernos.info, 39, 135-150. https://dx.doi.org/10.7764/cdi.39.970

Muñiz, C., Echeverría, M., Rodríguez-Estrada, A., y Díaz-Jiménez, O. (2016). Los hábitos comunicativos y su influencia en la sofisticación política ciudadana. Convergencia, 25(77), 1405-1435.

Murcia, N., Jaimes, S. S., Gómez, J. (2016). La práctica social como expresión de humanidad. Cinta de Moebio, 57, 257-274.

Natal, A., Benítez, M., \& Ortiz, G. (Eds.). (2014). Ciudadanía digital. Universidad Autónoma Metropolitana: Unidad Iztapalapa/División de Ciencias Sociales y Humanidades, Departamento de Antropología, Unidad Lerma/División de Ciencias Sociales y Humanidades, Departamento de Procesos Sociales. México.

Pérez, G., Carabaza, J., y Cuevas, B. (2013). Fuentes en Twitter: Análisis de las cuentas seguidas por los diarios Vanguardia, Diario de Coahuila y Zócalo de Saltillo. XX Anuario de investigación de la Comunicación, CONEICC. Gutiérrez, C. (coord.). CONEICC

Piñuel, J. L. y Gaytán, J. A. (1995). Metodología general. Conocimiento científico en investigación en la comunicación social. Síntesis.

Reguillo, R. (2018). Paisajes insurrectos. Jóvenes, redes y revueltas en el otoño civilizatorio. Cultura y Representaciones Sociales, 12(24), 433.

Ruiz, J. F. (2015). El impacto de los medios de comunicación sobre la definición del voto de los ciudadanos en Monterrey en las elecciones presidenciales de 2012. Revista Mexicana de Ciencias Politicas y Sociales, 60(225), 203-225.

Schuman, L., Blomberg, J., Orr, J., y Trigg, R. (1999). Reconstructing technologies as social practice. The American Behavioral Scientist, 43(3), 392-408.

Van Dijck, J. (2012). Tracing Twitter: the rise of a microblogging platform. International Journal of Media and Cultural Politics, 7(3), 333-348.

Verba, S., \& Almond, G. (1963). The civic culture. Political Attitudes and Democracy in Five Nations.

Yamamoto, M., Kushin, M., y Dalisay, F. (2015). Social media and mobiles as political mobilization forces for young adults: Examining the moderating role of online political expression in political participation. New Media \& Society, 17(6), 880-898. https://doi. org/10.1177/1461444813518390 